\title{
DEVELOPMENT OF STABLE NANOSUSPENSION LOADED ORAL FILMS OF GLIMEPIRIDE WITH IMPROVED BIOAVAILABILITY
}

\author{
VAISHALI KILOR ${ }^{* 1}$, NIDHI SAPKAL ${ }^{1}$, ANWAR DAUD ${ }^{2}$, SHRUTI HUMNE ${ }^{1}$, TUSHAR GUPTA ${ }^{1}$ \\ 1Gurunanak College of Pharmacy, Nagpur 440026, '2Zim Laboratories Ltd., Kalmeshwar, Nagpur 44002 \\ Email: v_kilor@yahoo.com
}

Received: 21 Dec 2016, Revised and Accepted: 02 Mar 2017

\section{ABSTRACT}

Objective: In the present work attempt has been made to stabilize optimized nanosuspensions of glimepiride by solidification using a novel Oral Thin Film (OTF) formulation.

Methods: Nanosuspensions were characterized for particle size, zeta potential as well as in vitro dissolution profile. As nanosuspensions are prone to destabilization by Ostwald's ripening or agglomeration/aggregation, OTF formulation as a novel approach for stabilization through solidification of optimized nanosuspension was attempted. OTF formulation method is a simple, easy and scalable method for the preparation of solid oral dosage form. Prepared formulations were evaluated for physicochemical parameters like folding endurance, disintegration time, tensile strength, in vitro drug release, in vivo bioavailability and stability.

Results: The mean particle size of the nanoparticles in OTF was found to be $57.2 \mathrm{~nm}$. It was observed from the results of in vivo bioavailability studies that high plasma drug concentrations(Cmax) were achieved for nanosuspension loaded OTF (NSOTF) i.e. 4900 ng/ml as compared to marketed oral formulation (Cmax-2900 ng/ml). Results of the stability studies indicated that nanosize of the particles was retained even after 3 mo of the study.

Conclusion: Therefore it can be concluded that OTF formulation has a potential for stabilization of nanosuspensions.

Keywords: Nanosuspension, Glimepiride, Stabilization, Solidification, In vivo studies

(C) 2016 The Authors. Published by Innovare Academic Sciences Pvt Ltd. This is an open access article under the CC BY license (http://creativecommons.org/licenses/by/4.0/) DOI: http://dx.doi.org/10.22159/ijap.2017v9i2.16714

\section{INTRODUCTION}

Nanotechnology-based techniques have been valuable in improving the solubility of poorly water soluble drugs [1]. Nanosuspensions in many cases have given good results in improving saturation solubility by increasing the surface area available for dissolution [2]. Various researchers have reported the development of nanosuspensions with high drug loading, increased dissolution and better bioavailability $[3,4]$. Studies have also been done to report non-toxic nature of these nanosuspensions [5]. Stability of nanosuspension is the major challenge associated with it. These are thermodynamically unstable and tend to show particle growth during storage. The change in particle size reverses all the advantages of solubility, dissolution and bioavailability. Various stabilizers like hydrophilic polymers [6-8] non-ionic or ionic surfactants and food proteins [9] have been used for stabilizing particle size of nanosuspensions, but the efficacy of these stabilizers is questionable upon dissolution, high temperature and $\mathrm{pH}$ changes. Further, as solidified form of nanosuspension is preferred over liquid form, significant work is reported on solidification techniques of nanosuspensions. These are solidified either using spray dryer $[10,11]$ or freeze dryer [12]. But it was observed that both of these techniques involve the control of multiple process parameters and still have problems of agglomeration during storage. Nanosuspensions have also been converted to hydrogels for the purpose of drug delivery and stability [13], but these hydrogels contain a large amount of water so may not be a system of choice for moisture sensitive drug.

Recently attempts have also been made to convert nanosuspensions into thin film formulation that offer both stability and ease of administration $[14,15]$. Thin films can further improve the solubility of drugs. Improved stability and bioavailability have been demonstrated $[16,17]$ for poorly water soluble drugs.

Glimepiride is one of the third generation sulfonyl urea used for the treatment of type 2 diabetes. It belongs to class II of Biopharmaceutical classification system showing poor aqueous solubility $(0.0082$ mmol) and high permeability [18]. Several attempts have been made to improve solubility of Glimepiride using approaches like solid dispersion [19] inclusion complexation [20] co-solvency [18] etc. But no systematic studies have been reported for enhancement of solubility using nanosuspension technique.

In the present work, an attempt has been made to prepare nanosuspension by using high shear homogenization to improve solubility of glimepiride and to improve the stability of glimepiride loaded nanosuspension by formulating into OTF.

\section{MATERIALS AND METHODS}

\section{Materials}

Glimepiride and Hydroxypropyl methylcellulose (HPMC) E 15 were obtained as gift samples from Zim Laboratories ltd., Nagpur, India. Sodium Dodecyl Sulfate (SDS) (Merck, Mumbai, India), PEG 400 (Loba Chemie Ltd. Mumbai, india) were procured commercially. All other chemicals and reagents were of analytical grade and were used without further purification.

Preparation of glimepiride nanosuspension by high shear homogenization

Glimepiride loaded nanosuspensions were prepared using high shear homogenization method. Stabilizer solution was prepared using 1\% HPMC and $12 \%$ SDS. The concentration of both of these stabilizers was selected during preliminary trials. The solution was stirred using high shear homogenizer (Remi-RQT-127 A/D, Mumbai, India) at $4000 \mathrm{rpm}$ for $15 \mathrm{~min}$. Glimepiride ( $0.4 \mathrm{~g}$ ) was then added to this solution while stirring and homogenization was continued at $8000 \mathrm{rpm}$ for $150 \mathrm{~min}$ at ambient conditions. To study the effect of stirring time on nanosuspension properties, above procedure was repeated by increasing homogenization time to $180 \mathrm{~min}$. Clear nanosuspensions so obtained were labeled as GNS1 and GNS2 and were stored in capped glass vials till further evaluation at room temperature.

Evaluation of glimepiride nanosuspensions

Particle size distribution and polydispersity index

Particle size was determined using Photon correlation spectroscopy 
(PCS) using Horiba Nanoparticle Analyser (Nanopartica SZ-100). This analysis yields the mean particle diameter (z-average), Polydispersity index and zeta potential at $25^{\circ} \mathrm{C}$.

\section{Percent drug content}

An aliquot $(0.1 \mathrm{ml})$ of nanosuspension was diluted to $10 \mathrm{ml}$ with methanol and filtered through $0.45 \mu \mathrm{m}$ filter paper. The sample was analyzed using UV Spectrophotometer (Shimadzu-1700, Japan) at $\lambda_{\max }$ of $233 \mathrm{~nm}$ using methanol as blank. Each sample was prepared and analyzed in triplicate.

\section{Saturation solubility studies}

Saturation solubility measurements were carried out for both pure drug and GNS1 and GNS2 using UV spectroscopy. Pure glimepiride $(10 \mathrm{mg}$ ) and nanosuspensions (GNS1 and GNS2) (equivalent to 10 mg Glimepiride), were weighed and separately introduced into 250 $\mathrm{ml}$ Stoppard conical flask containing $10 \mathrm{ml}$ of distilled water. The flask was sealed and placed in a rotary shaker at $37{ }^{\circ} \mathrm{C}$ and equilibrated for $2 \mathrm{~d}$. The contents were then filtered, and the suitably diluted samples were analyzed using UV-Visible spectrophotometer (Shimadzu-1700, Japan) at $233 \mathrm{~nm}$, against distilled water as a blank. Each sample was prepared and analyzed in triplicate.

\section{In Vitro dissolution studies}

In vitro drug release studies were performed on USP-Type II Basket apparatus at $75 \mathrm{rpm}$ in two dissolution media separately, $900 \mathrm{ml}$ of $\mathrm{pH} 1.2$ buffer and $900 \mathrm{ml}$ of $\mathrm{pH} 6.8$ phosphate buffer at $37.0 \pm 0.5^{\circ} \mathrm{C}$. TheHard gelatin capsules were filled with an accurately weighed quantity of pure glimepiride powder $(2 \mathrm{mg})$ and GNS1 and GNS2 (equivalent to $2 \mathrm{mg}$ of glimepiride) were used for dissolution. About $5 \mathrm{ml}$ of sample was withdrawn at predetermined time intervals and replaced with an equal quantity of fresh dissolution medium. These samples were filtered through $0.45-\mu \mathrm{m}$ Whatman filter paper and analyzed spectrophotometrically at $\lambda_{\max } 227 \mathrm{~nm}$. The in vitro dissolution testing studies were performed in triplicate.

\section{Preparation of glimepiride OTFs}

OTFs of optimized glimepiride nanosuspension were prepared by solvent casting method [16]. Accurately weighed quantities of HPMC E-15 (1 g) and PEG 400 (0.15 g) were added to glimepiride nanosuspension formulation GNS2 (equivalent to $40 \mathrm{mg}$ of glimepiride) and stirred using magnetic stirrer for $30 \mathrm{~min}$. The final mixture was then casted on a glass plate with the help of doctor's blade. It was dried in hot air oven for $2 \mathrm{~h}$ at $50{ }^{\circ} \mathrm{C}$. After drying, films were removed with the help of the sharp blade, cut in the suitable sizes containing glimepiride equivalent to $2 \mathrm{mg}$, packed in aluminum foil and kept in a desiccator till further evaluation. The films were labeled as NSOTF. The procedure was repeated for the formulation of OTFs of pure glimepiride powder and films so obtained were labeled as GOTF.

\section{Evaluation of OTFs}

\section{Thickness}

The thickness of the films was measured using the digital micrometre with an accuracy of $0.001 \mathrm{~mm}$. Thickness was measured for 10 different films and average thickness was determined.

\section{Tensile strength and percent elongation}

Tensile strength was determined using lab scale tensile strength to measure instrument. Films of dimension $3 \times 2 \mathrm{~cm}^{2}$ and free from physical imperfections were used for the study. The films were held between two clamps at a distance of $3 \mathrm{~cm}$. Films were pulled by the upper clamp at the rate of $5 \mathrm{~mm}$ per minute until it tears with the addition of weight at regular intervals. Measurements were done in triplicate for each batch. Tensile strength is the ratio of maximum stress applied to a point at which the film specimen breaks and can be computed from the applied force at rupture to the cross-sectional area of the fractured film as a mean of three measurements and described in the equation:

$$
\text { Tensile strength }=\frac{\text { Force at break }(N)}{\text { Initial cross sectional area }(\mathrm{mm})} X 100
$$

Percent elongation was calculated using the following formula:

$$
\text { Percent elongation }=\frac{\text { Inerease in length }}{\text { Original longth }} \times 100
$$

\section{Folding endurance}

Folding endurance is determined by repeated folding of the strip at the same place till the strip breaks. The number of times the film is folded without breaking is computed as the folding endurance value. The folding endurance studies were performed for six strips for each batch i.e. NSOTF and GOTF.

\section{Percent moisture absorption}

The moisture absorption capacity of the films was determined by exposing the pre-weighed films to $75 \pm 2 \%$ relative humidity (created by using a saturated solution of $\mathrm{NaCl}$ ) in a desiccator at room temperature. Film samples were weighed at predetermined intervals until constant weight was achieved. Percentage moisture uptake was calculated as the percentage of the difference between the final and initial weight with respect to the final weight as per the following formula:

$$
\% \text { Moisture absorption }=\frac{\text { Final weight }- \text { Initial weight }}{\text { Initial weight }} \times 10 \mathrm{C}
$$

\section{Disintegration test}

Disintegration test was performed in the USP Disintegration apparatus. Simulated salivary fluid ( $\mathrm{pH}$ 6.8) was used as the medium. The films were placed in the tubes of the container, and the disks were placed over it. The average disintegration time of six films was noted for both the batches.

\section{Percent drug content}

Six strips from each batch were picked randomly and were weighed individually. Each film was sonicated with methanol $(10 \mathrm{ml})$ for $2 \mathrm{~h}$. The final mixture $(1 \mathrm{ml})$ was filtered through 0.45 filter and suitably diluted with methanol. The UV absorbance of the solution was measured at $227 \mathrm{~nm}$, using methanol as blank. An average of six films was reported as average drug content.

\section{In vitro dissolution studies}

In vitro dissolution studies of NSOTF (containing nanoparticles) and GOTF (containing pure Glimepiride) were performed in $900 \mathrm{ml}$ of $\mathrm{pH} 1.2 \mathrm{HCl}$ buffer at $37 \pm 0.5^{\circ} \mathrm{C}$ using USP Type II (Paddle) Dissolution apparatus at $75 \mathrm{rpm}$. Aliquots $(5 \mathrm{ml})$ were collected at specific time intervals and the volume was made up by adding fresh dissolution medium. Glimepiride was determined spectrophotometrically (UV 1800, Shimadzu, Japan) at $\lambda_{\max } 227 \mathrm{~nm}$. Studies were repeated using $900 \mathrm{ml}$ of $\mathrm{pH} 6.8$ phosphate buffer as a dissolution medium also. Results are expressed as the mean of three determinations. Dissolution studies of glimepiride marketed tablets were also carried out using both the dissolution media for comparison purpose.

\section{In vivo studies of glimepiride OTF}

The protocol of animal study was approved by the institutional animal ethics committee. Rabbits of either sex (Weight, 2-3 kg) were fasted overnight before the studies. The rabbits were randomly divided into three groups of six animals each. The Group I was given GOTF, Group II, NSOTF and Group III were given marketed tablet. Both the films and a tablet containing $2 \mathrm{mg}$ of glimepiride were dispersed separately in $5 \mathrm{ml}$ of water, and the dispersion was carefully fed to animals using glass syringe without needle ensuring the complete administration. Blood samples for pharmacokinetic analysis were withdrawn at intervals of $0,1 \mathrm{~h}, 2 \mathrm{~h}, 4 \mathrm{~h}, 6 \mathrm{~h}, 8 \mathrm{~h}$ and $12 \mathrm{~h}$ from the marginal ear vein. Blood samples were collected in heparinized tubes and centrifuged for $10 \mathrm{~min}$ at 5,000 rpm in a refrigerated centrifuge. The plasma samples were then stored at $-4{ }^{\circ} \mathrm{C}$ till further analysis.

\section{Plasma analysis}

About $2 \mathrm{ml}$ of chloroform was added to plasma $(0.5 \mathrm{ml})$ and vortexed for $5 \mathrm{~min}$. The mixture was centrifuged at $5000 \mathrm{rpm}$ for 10 min; the chloroform layer was then evaporated to dryness. The residue so obtained was reconstituted in $2 \mathrm{~m} 1$ of the mobile phase, 
filtered and was analysed for glimepiride content using HPLC (Agilent Technologies) using following conditions:

Column: Eclipse XDB, C18

Mobile Phase: Acetonitrile: Water: : 90:10

Flow Rate: $1 \mathrm{ml} / \mathrm{min}$

Sample Size: $20 \mu \mathrm{l}$

UV Detection wavelength: $228 \mathrm{~nm}$

\section{Stability studies}

Both NSOTF and GOTF formulations were subjected to accelerated stability studies as per ICH guidelines at $45{ }^{\circ} \mathrm{C}, 75 \%$ RH. Film samples were evaluated after 1,2 and 3 mo for disintegration time, mean particle size and dissolution profile to study the stability of nanosuspension loaded OTFs.

\section{RESULTS AND DISCUSSION}

\section{Evaluation of glimepiride nanosuspension}

Attempts to improve solubility of poorly soluble glimepiride has been made several times, but no report in literature is available for the nanosuspension formulation of glimepiride for improving its solubility. Nanosuspensions of glimepiride were prepared using high shear homogenizer at a constant speed for 150 to $180 \mathrm{~min}$. The aim was to study the effect of homogenization time on the characteristics of nanosuspensions. The particle size, polydispersity index and zeta potential are given in table 1 . The table shows that homogenization time has the profound impact on the particle size and polydispersity index. Increasing the homoginization time reduces the mean particle size from $160.6 \mathrm{~nm}$ to $98.1 \mathrm{~nm}$. Polydispersity Index was found to be 0.042 for GNS1 and 0.562 for GNS2. Polydispersity index is the indicator of uniformity in particle size distribution. The obtained value indicated the uniformity in particle size distribution in both the formulations. Fig. 1 depicts the particle size distribution of GNS2. From table 2 it was seen that percent drug content of GNS1 and GNS2 was $92.95 \%$ and $95.42 \%$ respectively indicating that increasing homogenization time reduces the particle size and hence increases the drug content in the clear fraction of nanosuspension. Because of higher drug content, saturation solubility of GNS2 is more than GNS1.

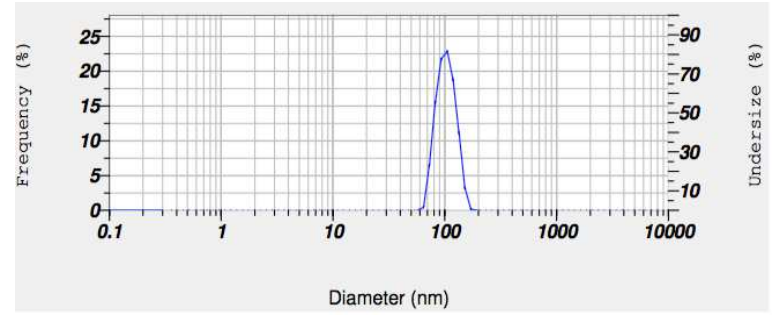

Fig. 1: Particle size distribution of GNS2

Table 1: Particle size, polydispersity index and zeta potential of glimepiride loaded nanosuspensions

\begin{tabular}{llll}
\hline Formulation code & Mean particle size (nm) & Polydispersity index & Zeta potential (mV) \\
\hline GNS1 & $160.6 \pm 84.2$ & 0.042 & -99.6 \\
GNS2 & $98.1 \pm 2.2$ & 0.562 & -18.2 \\
\hline
\end{tabular}

GNS-glimepiride nanosuspension, mean $\pm \mathrm{SD}(\mathrm{n}=3)$

Table 2: Percent drug content and saturation solubility of glimepiride loaded nanosuspensions

\begin{tabular}{lll}
\hline Formulation code & *Percent drug content & *Saturation solubility $(\mathbf{m c g} / \mathbf{m l})$ \\
\hline Glimepiride & -- & \\
GNS1 & $92.95 \pm 0.25$ & $669.57 \pm 1.47$ \\
GNS2 & $95.428 \pm 1.09$ & $814.17 \pm 0.15$ \\
\hline
\end{tabular}

$*$ mean $\pm \mathrm{SD}(\mathrm{n}=3)$

\section{In vitro dissolution studies of glimepiride nanosuspensions}

In vitro dissolution studies revealed that nanosuspension formulations resulted in a dramatic increment in the glimepiride solubility. Both GNS1 and GNS2 showed almost complete drug release within 10 min whereas pure glimepiride dissolved only up to $25 \%$ in first 10 min. GNS1 and GNS2 showed a similar release profile in pH 1.2 and $\mathrm{pH} 6.8$ phosphate buffer. This shows that solubility of glimepiride loaded nanosuspension is independent of the $\mathrm{pH}$ of the surrounding medium. In the earlier studies conducted on glimepiride 40 to $75 \%$ improvement in dissolution rate was observed in case of solid dispersions prepared using PVP k-30 as compared to pure drug whereas solid dispersions prepared using guar gum could release only $30 \%$ drug within $10 \mathrm{~min}$. [21,22]. Therefore it can be said that formulating nanosuspensions of glimepiride by simple high shear homogenization method using hydrophilic polymer HPMC E-15 which acts as a steric stabilizer could enhance dissolution rate significantly [23]

The increase in dissolution profile of GNS2 may be due to an increase in the stirring times during preparation as compared to their respective nanosuspension formulations, which resulted in smaller particle sizes. As the size decreases, the effective increase in particle surface area results in an increase in dissolution velocity according to the Nernst Brunner-Noyes Whitney equation [24].
A large surface area-to-volume and higher apparent saturation solubility of nanoparticles pronouncedly enhanced the dissolution rate. Finally, the increase in surface wetting by the surfactants in the nanosuspension formulations most likely results in further enhancement of the dissolution rates compared to the pure drug particles.

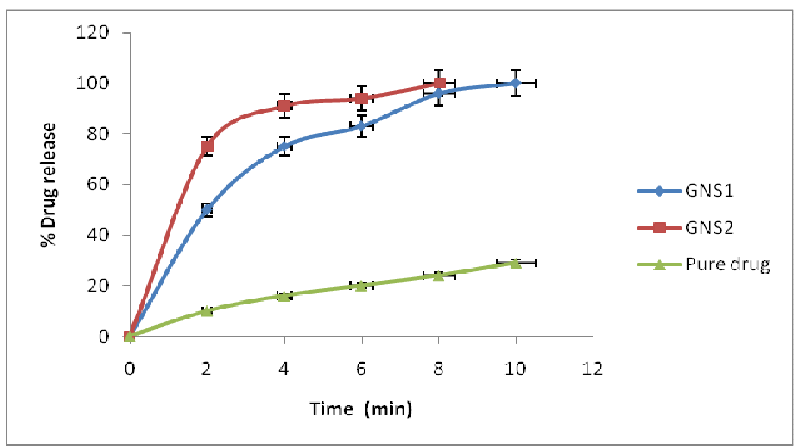

Fig. 3: In vitro dissolution profiles of GNS 1, GNS 2 and pure glimepiride inpH $1.2 \mathrm{HCl}$ buffer 


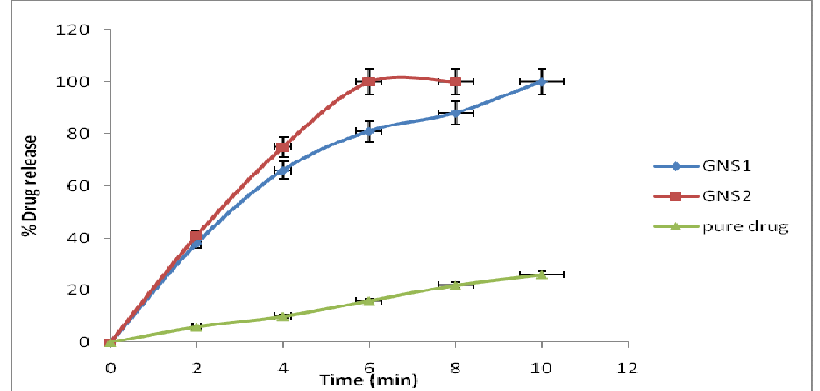

Fig. 4: In vitro dissolution profiles of GNS 1, GNS 2 and pure glimepiride in $\mathrm{pH} 6.8$ phosphate buffer

Solidification of nanosuspensions in various ways like spray drying, freeze drying has been explored by many researchers for two reasons, one is to formulate the nanosuspension in a suitable unit dosage form and second is to improve their stability. The biggest draw- back of nanoparticles is they tend to form aggregates upon standing. Many researchers have used various types of stabilisers in optimized concentrations to improve the stability of nanosuspensions [25]. In the present work, an attempt was made by converting nanosuspensions into thin flat films. The film forming polymer and plasticizers were added to the dispersion containing nanosuspension. The high viscosity of the final dispersion prevents the nano suspended particles from forming aggregates. After casting and drying the stability of the particles is ensured better [26].

Table 3 shows the comparison of physicochemical characterization of pure glimepiride films (GOTF) and nanosuspension loaded glimepiride films (NSOTF). Both the films were found to be nonsticky, translucent with a smooth surface. NSOTF showed good mechanical properties. All the physicochemical properties were found to be comparable in both the films. This may be because there are minor differences in the composition of both the films. The assay (\% drug content) of nanosuspension loaded films was within the IP limits because of better mixing efficiencies are achieved in smaller and uniformly suspended particles.

Table 3: Physicomechanical characterization of NSOTF

\begin{tabular}{llll}
\hline S. No. & Parameters & Formulation code & \\
\cline { 3 - 4 } & & GOTF & NSOTF \\
\hline 1 & Appearance & *Translucent film with smooth surface & ${ }^{*}$ Translucent film with smooth surface \\
2 & Average weight & $45.66 \pm 0.32$ & $47.08 \pm 1.10$ \\
3 & Thickness $(\mathrm{mm})$ & $0.136 \pm 0.5$ & $0.141 \pm 0.032$ \\
4 & Tensile strength $\left(\mathrm{N} / \mathrm{mm}^{2}\right)$ & $7.83 \pm 0.17$ & $7.95 \pm 0.12$ \\
5 & Percent Elongation & $16.98 \pm 0.58$ & $18.25 \pm 1.68$ \\
6 & Folding endurance & $15 \pm 3$ & $20 \pm 2$ \\
7 & (\%) Moisture Absorption & $8.85 \pm 0.23$ & $9.35 \pm 0.14$ \\
8 & Disintegration Time (sec) & $29.15 \pm 1.12$ & $27.84 \pm 0.95$ \\
9 & Drug content (\%) & 95.480 & 98.030 \\
\hline
\end{tabular}

* mean $\pm \mathrm{SD}(\mathrm{n}=3)$

Fig. 5 and 6 gives comparative in vitro dissolution profile of GOTF, NSOTF and marketed tablets in pH 1.2 and 6.8. Fig. reveals that marketed tablets showed less than $30 \%$ dissolution in both the media. An important finding of this work as that OTFs improve dissolution of the poorly soluble drugs. NSOTF showed more than $95 \%$ dissolution in both the dissolution media.

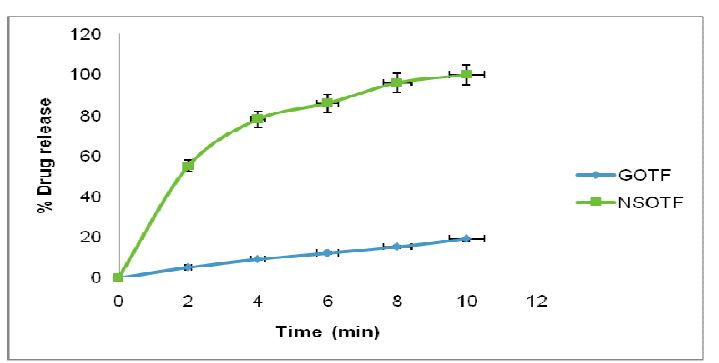

Fig. 5: In vitro dissolution profile of GOTF and NSOTF in pH 1.2 HCl buffer

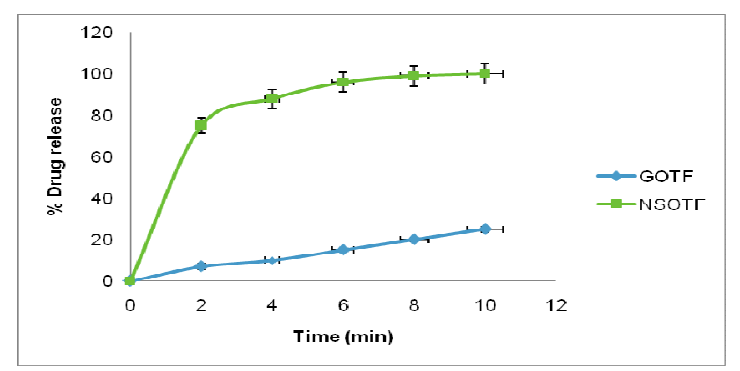

Fig. 6: Comparative in vitro dissolution profile of GOTF and NSOTF in pH 6.8 phosphate buffer
In vivo pharmacokinetic studies of glimepiride nanosuspensions

The differences in between the formulations were more evident during in vivo studies (table 4). Plasma concentration of glimepiride increased more rapidly in case of NSOTF than in GOTF and marketed tablets. The t-max was $2 \mathrm{~h}$ and Cmax was $4900 \mathrm{ng} / \mathrm{ml}$ for NSOTF which was highest among all the three formulations, whereas marketed tablet showed lowest Cmax (2900ng/ml). Area under the curve (AUC) values calculated for $12 \mathrm{~h}$ from the plasma concentration of glimepiride by Trapezoidal rule. The values have significantly increased from $22650 \mathrm{ng}$. $\mathrm{h} / \mathrm{ml}$ to $27800 \mathrm{ng}$. $\mathrm{h} / \mathrm{ml}$ by converting glimepiride into OTFs. This effect is due to the complete dissolution of glimepiride in the GI tract. Formulation NSOTF showed the highest AUC (37444 ng. $\mathrm{h} / \mathrm{ml}$ ) i.e. significant enhancement in bioavailability. Thus nanosuspension loaded OTFsis a better and novel option for improvement of bioavailability with a faster onset of action of poorly soluble drugs.

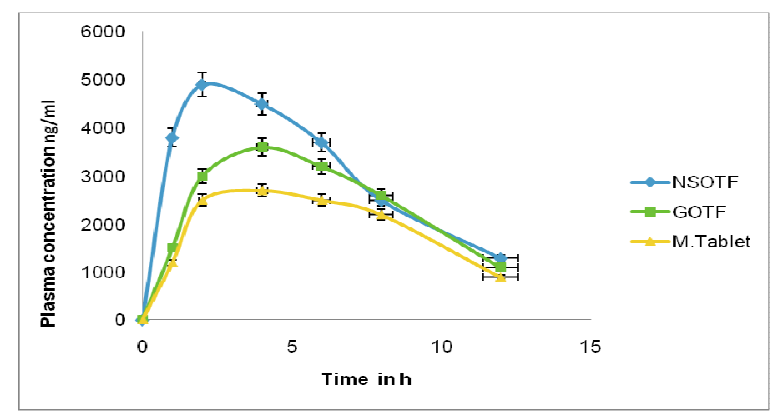

Fig. 7: In vivo drug release profiles of GOTF, NSOTF and marketed tablet 
Table 4: Pharmacokinetic parameters of GOTF, NSOTF and marketed tablet

\begin{tabular}{lllll}
\hline S. No. & Parameter & NSOTF & GOTF & marketed tablet \\
\hline 1 & Cmax (ng/ml) & 4900 & 3600 & 2900 \\
2 & tmax (h) & $2 \mathrm{~h}$ & $4 \mathrm{~h}$ & $4 \mathrm{~h}$ \\
3 & AUC $(\mathrm{ng} . \mathrm{h} / \mathrm{ml})$ & 37444 & 27800 & 22650 \\
\hline
\end{tabular}

\section{Stability studies}

From stability studies of OTFs (table 5) it was observed that the physicochemical properties were retained within the specified limits i.e. films were transparent visually, and percent moisture absorption increased from 9.35 to $10.27 \%$ which did not affect the ease of handling of the film as well as folding endurance was also within limits. Film disintegrated within $28 \mathrm{sec}$ and $\%$ drug content decreased from $98.03 \%$ to $96.78 \%$ within 3 mo at $40{ }^{\circ} \mathrm{C} / 75 \%$ RH. There was no significant difference in \% dissolution of the drug in 1.2 as well as 6.8
pH (fig. 8 and fig. 9).

The particle size of the drug dispersed in the OTF was measured using Horiba Nanoparticle Analyzer at $25^{\circ} \mathrm{C}$ and scattering angle 90 ${ }^{\circ} \mathrm{C}$. From table 6 it can be seen that particle size of the drug reduced from $98.1 \mathrm{~nm}$ to $57.2 \mathrm{~nm}$. There was no significant change in the zeta potential. This may be because of the presence of HPMC E-15 in the film base which helps in preventing crystallisation of drug particles as well as causes drug solubilization $[27,28]$ along with other excipients used as a film base like PEG 400.

Table 5: Physicochemical parameters of NSOTF during stability studies

\begin{tabular}{llll}
\hline S. No. & Parameters & *Initial (at 0 mo) & *after 3 mo \\
\cline { 2 - 4 } & & NSOTF & NSOTF \\
\hline 1 & Appearance & Transparent & $45.085 \pm 1.10$ \\
2 & Weight variation $(\mathrm{mg})$ & $0.131 \pm 0.032$ & $44.059 \pm 1.23$ \\
3 & Thickness $(\mathrm{mm})$ & $7.85 \pm 0.12$ & $0.130 \pm 0.25$ \\
4 & Tensile strength $\left(\mathrm{N} / \mathrm{mm}^{2}\right)$ & $17.25 \pm 1.68$ & $8.48 \pm 0.22$ \\
5 & $\%$ Elongation & $20 \pm 2$ & $19.18 \pm 1.02$ \\
6 & Folding endurance & $8.35 \pm 0.14$ & $25 \pm 2$ \\
7 & (\%) moisture absorption & $27.84 \pm 0.95$ & $10.27 \pm 1.47$ \\
8 & Disintegration (sec) & $6-7$ & $26.25 \pm 0.52$ \\
9 & Surface pH & 98.030 & $6-7$ \\
10 & Drug content $(\%)$ & 96.78 & \\
\hline
\end{tabular}

${ }^{*}$ mean $\pm \mathrm{SD}(\mathrm{n}=3)$

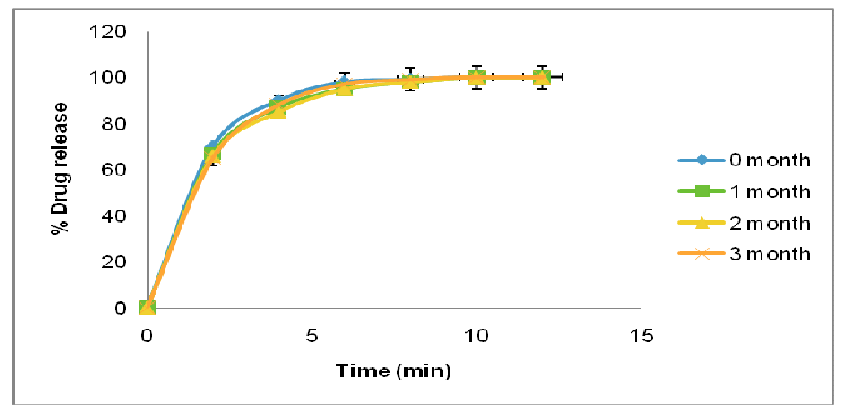

Fig. 8: In vitro dissolution studies on NSOTF in pH 1.2 buffer

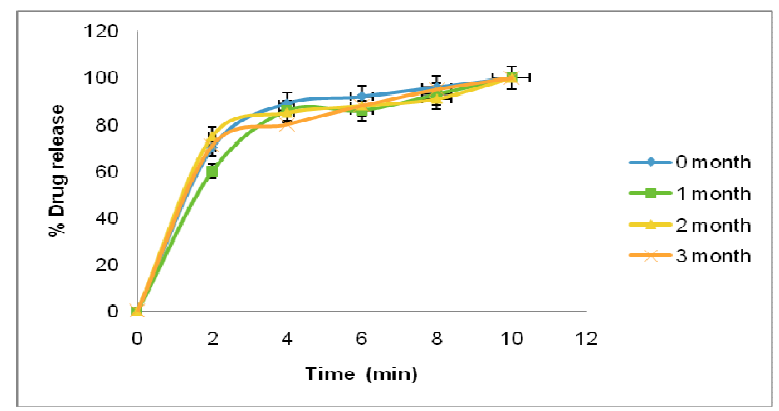

Fig. 9: In vitro dissolution studies on NSOTF in pH 6.8 buffer

Table 6: Particle size and zeta potential of NSOTF (Nanosuspension OTF of glimepiride)

\begin{tabular}{lllll}
\hline Formulation & Particle size at 0 mo & Particle size after 3 mo & Zeta potential at 0 mo & Zeta potential after 3 mo \\
\hline NSOTF & $98.1 \mathrm{~nm}$ & $57.2 \mathrm{~nm}$ & -18.2 & -20.6 \\
\hline
\end{tabular}

$*_{\text {mean } \pm S D}(\mathrm{n}=3)$

\section{CONCLUSION}

From the above findings, it can be concluded that formulation of nanosuspension loaded OTF can be a novel option for stabilisation as well as solidification of nanosuspensions. In this work Simple high shear homogenization method led to the formation of nanosuspension with significant improvement in dissolution rate. Transformation of nanosuspension into OTF stabilized nanoparticles by retaining particle size in nano range. Improved bioavailability of the drug in OTF formulation was evident from high Cmax and tmax values. Thus OTF formulation as a solidification technique can be very promising for stabilization of nanosuspensions.

\section{ACKNOWLEDGEMENT}

Authors would like to acknowledge Dr. A. M. Ittadwar, Principal, Gurunanak College of Pharmacy, Nagpur for providing infrastructural facilities to carry out the research work. We are also thankful to Dr. Vipin Dhote, Principal VNS Institute of pharmacy, Bhopal for providing facilities for carrying out particle size analysis using Horiba Nanosizer.

\section{CONFLICTS OF INTERESTS}

Declare none 


\section{REFERENCES}

1. Cooper ER. Nanoparticles: a personal experience for formulating poorly water-soluble drugs. J Controlled Release 2010;141:300-2.

2. Wang Y, Zhang L, Wang Q Zhang D. Stability issue of nanosuspensions in drug delivery. J Controlled Release 2013;172:1126-41.

3. Laxmi P, Ashwinikumar G. Nanosuspension technology: a review. Int J Pharm Pharm Sci 2010;(2 Suppl 4):35-40.

4. Baek I, Kim J, Eun-Sol Ha, Gwang-Ho Choo. Dissolution and oral absorption of pranlukast nanosuspensions stabilized by hydroxypropylmethylcellulose. Int J Biol Macromol 2014;67:53-7.

5. Baumgartner R, Birgit J Teubl, Carolin Tetyczka, Roblegg E. Ratinal design and characterization of a nanosuspension for intraoral administration considering physiological conditions. J Pharm Sci 2016;105:257-67.

6. Singh SK, Srinivasan KK, Gowthamarajan K, Singare DS, Prakash D, Gaikwad NB. Investigation of preparation parameters of nanosuspension by top-down media milling to improve the dissolution of poorly water-soluble glyburide. Eur J Pharm Biopharm 2011;71:441-6.

7. Ghosh S, Bose R, Vippagunta F. Harmon. Nanosuspension for improving the bioavailability of a poorly soluble drug and screening of stabilizing agents to inhibit crystal growth. Int J Pharm 2011;409:260-8

8. Rachmawati H, Al Shaal L, Muller RH, Keck CM. Development of curcumin nanocrystal: physical aspects. J Pharm Sci 2013;102;204-14.

9. He W, Lu Y, Qi J, Chen L, Hu F, Wu W. Food proteins as novel nanosuspension stabilizers for poorly water-soluble drugs. Int J Pharm 2013:441:269-78.

10. Gao YA, Qian SA, Zhang JJ. Physicochemical and pharmacokinetic characterization of a spray-dried cefpodoxime proxetil nanosuspension. Chem Pharm Bull 2010;58:912-7.

11. Mezhericher M, Naumann M, Peglow M, Levy A, Tsotsas E, Borde I. Continuous species transport and population balance models for first drying stage of nanosuspension droplets. Chem Eng J 2012;210:120-35.

12. Eerdenbrugh VB, Froyen L, Humbeeck JV, Martens JA, Augustijns P, Mooter G. Drying of crystalline drug nanosuspensions-the importance of surface hydrophobicity on dissolution behaviour upon redispersion. Eur J Pharm Sci 2008;35:127-35.

13. Gupta S, Samanta MK, Raichur AM. Dual-drug delivery system based on in situ gel-forming nanosuspension of forskolin to enhance antiglaucoma efficacy. AAPS PharmSciTech 2010;11:322-35.

14. Chonkar AD, Venkat Rao JRS, Managuli RS, S Mutalik. Development of fast dissolving oral films containing lercanidipine hcl nanoparticles in the semicrystalline polymeric matrix for enhanced dissolution and ex vivo permeation. Eur J Pharm Biopharm 2016;103:179-91.

15. Steiner D, Finke JH, Kwade A. Efficient production of nanoparticle-loaded orodispersible films by process integration in a stirred media mill. Int J Pharm 2016;511:804-13.
16. Shen B, Shen C, Yuan X. Development and characterization of an orodispersible film containing drug nanoparticles. Eur J Pharm Biopharm 2013;85:1348-56.

17. Figueroaa L, Bhakaya A, Jackeline I, Rozob J. Preparation and characterization of hydroxypropyl methyl cellulose films containing stable BCS Class II drug nanoparticles for pharmaceutical applications. Int J Pharm 2012;423:496-508.

18. Bhanja SC, Shafeeque M, Sudhakar M. Mucoadhesive buccal tablets of glimepiride-formulation and evaluation. Int J Pharm Pharm Sci 2013;4:502-10.

19. Chaudhari MD, Sonawane RO, Zawar L, Nayak S, Bari SB. Solubility and dissolution enhancement of poorly water-soluble glimepiride by using solid dispersion technique. Int J Pharm Pharm Sci 2012;(4, Suppl 5):534-9.

20. Ammar HO, Salama HA, Ghorab M, Mahmoud AA. Formulation and biological evaluation of glimepiride-cyclodextrin-polymer systems. Int J Pharm 2006;309:129-38.

21. Nagpal M, Rajera R, Nagpal K, Rakha P, Singh S, Mishra D. Dissolution enhancement of glimepiride using modified gum karaya as a carrier. Int J Pharm Investigation 2012;2:42-7.

22. Okonogi S, Puttipipatkhachorn S. Dissolution improvement of igh drug-loaded solid dispersion. AAPS PharmSciTech 2006; 7:1-6.

23. Thakar D, Bharadia P, Pandya V. Enhancement of solubility of poorly water soluble antihypertensive drug by the nanosizing approach. J Pharm Bioall Sci 2012;4(Suppl 1):S40-S41.

24. Muhammad I, Sumeira R, Quratulain B. Orally disintegrating films: a modern expansion in drug delivery system. Saudi Pharm J 2016;24:537-46.

25. Esfandia E, Ramezania V, Vatanaraa A. Clarithromycin dissolution enhancement by preparation of aqueous nanosuspensions using sonoprecipitation technique. Iranian J Pharma Res 2014;13:809-18.

26. Ghosh I, Bose S, Vippagunta R, Harmon F. Nanosuspension for improving the bioavailability of a poorly soluble drug and screening of stabilizing agents to inhibit crystal growth. Int J Pharm 2011;409:260-8.

27. Sievens-Figueroa L, Bhakay A, Jackeline I. Preparation and characterization of hydroxypropyl methyl cellulose films containing stable BCS Class II drug nanoparticles for pharmaceutical applications. Int J Pharm 2012;423:496-508.

28. Yokoi Y, Yonemochi E, Terada K. Effects of sugar ester and hydroxypropyl methylcellulose on the physicochemical stability of amorphous cefditoren pivoxil in aqueous suspension. Int J Pharm 2005;290:91-9.

\section{How to cite this article}

- Vaishali Kilor, Nidhi Sapkal, Anwar Daud, Shruti Humne, Tushar Gupta. Development of stable nanosuspension loaded oral films of glimepiride with Improved bioavailability. Int J Appl Pharm 2017;9(2):28-33. 